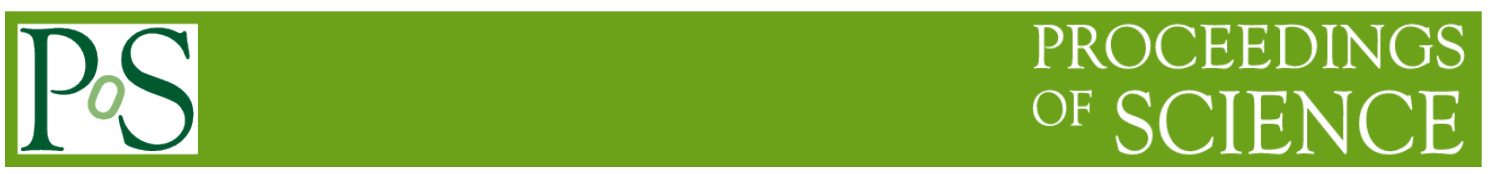

\title{
The mechanical structure and deployment procedure of the KM3NeT detection unit.
}

\author{
P.Kooijman ${ }^{1}$ (on behalf of the KM3NeT collaboration) \\ University of Amsterdam/nikhef \\ Science Park 105, 1098XG Amsterdam, The Netherlands \\ E-mail: h84@nikhef.nI
}

In this paper we provide a detailed description of the mechanical structure of the $750 \mathrm{~m}$ high KM3NeT detection unit. The choices made for the different materials and their behaviour under the loads expected during deployment and during the lifetime of the experiment will be discussed, as will the motion of the unit under influence of the sea currents. The unique method of deployment, which entails unfurling of the unit from the seabed using a purpose built launcher, will be described.

The 34th International Cosmic Ray Conference

30 July- 6 August, 2015

The Hague, The Netherlands

${ }^{1}$ Speaker 


\section{Introduction}

$\mathrm{KM} 3 \mathrm{NeT}$ is the next generation neutrino telescope, currently being constructed in the Mediterranean Sea. The first, ongoing and fully funded, phase of the project is the construction and deployment of 30 detection units. Each unit comprises 18 Digital Optical Modules [1] arranged on a vertical string-like structure. Plans for the second phase, for which funding is presently being sought, calls for the construction of a further 300 detection units. About two thirds of these are full size, $750 \mathrm{~m}$ tall units to be deployed near Capo Passero off the eastern coast of Sicily, comprising the neutrino astronomy (ARCA) part of the project. One third of the units will be about $200 \mathrm{~m}$ tall and be deployed off the coast of Toulon for the measurement of the neutrino mass hierarchy (ORCA). This paper describes the mechanical structure of the full length detection unit and the method for its deployment. All details given also hold for the shorter units except for the distance between optical modules.Introduction

\section{Design of the Detection Unit}

For ARCA simulations have shown that the optimal configuration of a detection unit comprises 18 digital optical modules (DOM) distributed vertically with an inter-DOM distance of between 35 and $40 \mathrm{~m}$ resulting in a structure of approximately $750 \mathrm{~m}$ height. These units are to be placed with a horizontal separation of $100 \mathrm{~m}$ at a depth of about $3200 \mathrm{~m}$ on the bottom of the Mediterranean Sea. The height and relatively small mutual distances together with the prevailing currents in the place of deployment set stringent requirements on the hydrodynamic behaviour of the detection unit. Currents in the deep Mediterranean Sea occasionally reach values of $0.3 \mathrm{~m} / \mathrm{s}$. At these values the top of the detection unit should not deflect more than the inter-unit distance. To avoid excessive added buoyancy at the top of the unit and a commensurately large mass anchor at the bottom of the unit, the design attempts to reduce the hydrodynamic drag of the vertical structure as much as possible. The 18 DOMs form an irreducible contribution to the hydrodynamic drag and so the design requires the mechanical and electro-optical connections to present as small as possible cross sectional area to the sea currents. The final design of the unit is shown in figure 1.

The mechanical connection is provided by two, $4 \mathrm{~mm}$ thick, ropes of woven Dyneema ${ }^{\circledR}$ DM20 fibre [2]. The fibre strands are made, by $\mathrm{DSM}^{2}$, of Ultra-High Molecular Weight Polyethylene and so have very high resistance to aging in sea water. The DM20 type is a modification of the SK78 rope manufactured to have superior characteristics in terms of long term creep. The $4 \mathrm{~mm}$ thick rope has a yield strength of $12 \mathrm{kN}$. The ropes run the full length of the detection unit and at the 35 to $40 \mathrm{~m}$ intervals the DOMs are attached to the ropes. In between the DOMs three spacers are fixed at regular intervals between the ropes to add torsional stability to the structure.

Figure 2 shows the detail of the connection. One high density polyethylene block, containing threaded titanium inserts, is connected to the Titanium collar that surrounds the DOM. The rope runs through a groove in the block, separated from it by a natural rubber strip. A titanium pin passes through the rope (without severing the fibres). A second block also

${ }^{2}$ DSM, P.O. Box 6500, 6401JH Heerlen, Netherlands 


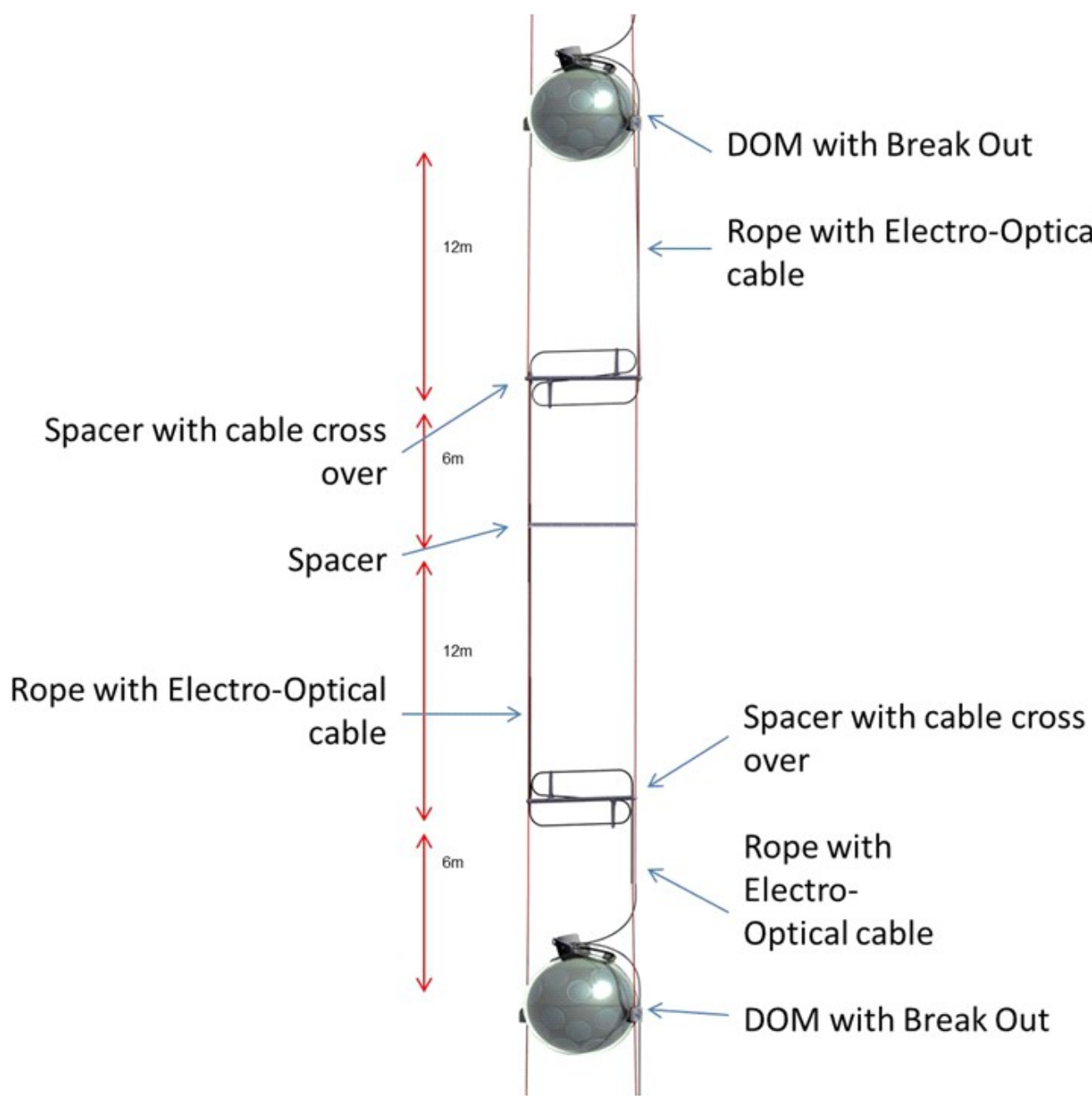

Figure 1:The mechanical design of the KM3NeT detection unit. For clarity only the section between two DOMs is shown. This structure is repeated up the unit. The spacers are for torsional stability and allow for the electro-optical cable to swap to the opposite rope and for the required extra cable length to be positioned.

containing a groove with a rubber strip is bolted over the rope to provide a $60 \mathrm{~mm}$ long friction connection, with the titanium pin as safety in case of sharp jolts during deployment. The centre of gravity of the DOM is situated marginally above the equator. Therefore the connection to the rope is connected $40 \mathrm{~mm}$ below the equator so that the righting moment due to the $420 \mathrm{~N}$ of buoyancy is always larger than the tipping moment due to the mass of $28 \mathrm{~kg}$ of the DOM. The length of the rope connection also aids avoiding forward backward tipping.

The electro-optical connection between the DOMs is made using a custom made pressure balanced cable. Two copper wires and fibres are strung through a thin polyethylene hose. A compromise was sought between drag caused by the cross sectional area and the ease of stringing of the fibres through the hose. Several (standard) sizes have been tried but finally the optimal size has been found to be a (custom made) $7 \mathrm{~mm}$ diameter with $1 \mathrm{~mm}$ wall thickness. The cable is made out of $40 \mathrm{~m}$ sections connected via a Breakout Box $(\mathrm{BoB})$. These provide the possibility to extract one fibre and two copper power cables to the DOM. 

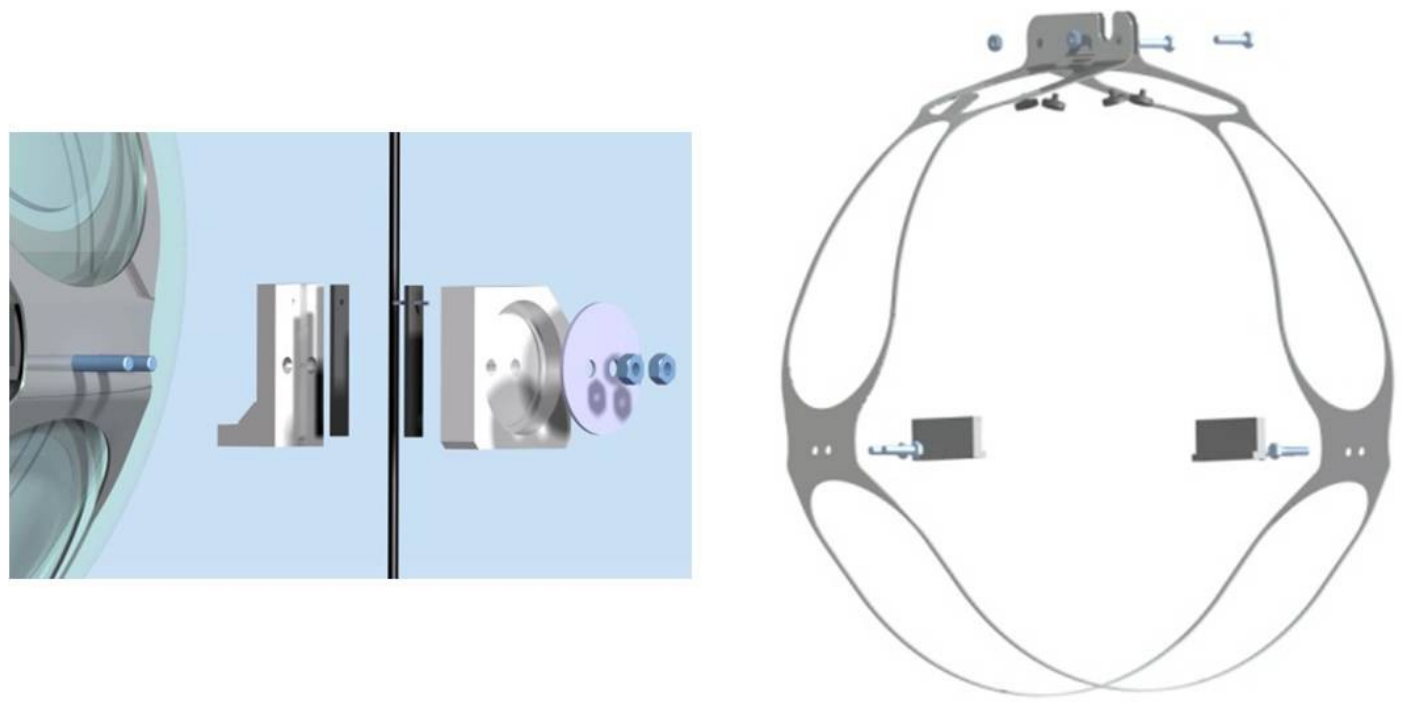

Figure 2: Detail of the connection of the rope to the DOM. Left the friction connection is shown and right gives the detail of the Titanium collar.

The first section does not have a DOM connected so the first DOM is situated at approximately $80 \mathrm{~m}$ above the sea bed. Each $\mathrm{BoB}$ also contains a DC/DC converter to step down the voltage from the $400 \mathrm{~V}$ of the cable to the $12 \mathrm{~V}$ required by the DOM. Twelve fibers run the full length of the cable an additional 12 fibers run from the bottom of the string to the ninth DOM. This arrangement gives one fiber per DOM with three spares in each set of nine DOMs. The cable and BoBs are filled with oil (MIDEL $7131[3]^{3}$ )so that all internal elements, fibers and DC/DC converters run at ambient pressure (up to $32 \mathrm{MPa}$ ). The BoBs are mounted on the top of the DOM fixed to the titanium collar. A short section of hose runs from the BoB to the penetrator, manufactured by $\mathrm{Pave}^{4}$, installed in the upper glass hemisphere of the DOM.

Additional buoyancy is provided by a cylindrical buoy, manufactured from syntactic foam inside a fibreglass-resin skin, manufactured by BMTI ${ }^{5}$. The size is $420 \mathrm{~mm}$ diameter by 1700 $\mathrm{mm}$ high and provides a buoyancy of $1350 \mathrm{~N}$. Each DOM provides a buoyancy of $140 \mathrm{~N}$. This leads to the overall buoyancy of the Detection unit of just under $4000 \mathrm{~N}$. The oil filled cable and the Dyneema ${ }^{\circledR}$ ropes are essentially neutrally buoyant.

Figure 3 shows the shape of the detection unit for different current speeds. It should be noted that currents of above $12 \mathrm{~cm} / \mathrm{s}$ are observed less than $5 \%$ of the time at the deployment site. For the detection unit for ORCA the inter-DOM distance is assumed to be between $6 \mathrm{~m}$ and $12 \mathrm{~m}$. It is seen that the deviation at the top of the unit is very close to the required inter-string distance of $100 \mathrm{~m}$ (ARCA) and $20 \mathrm{~m}$ (ORCA).

\footnotetext{
${ }^{3}$ M\&I Materials Ltd., Hibernia Way, Trafford Park, Manchester M32 0ZD, United Kingdom

${ }^{4}$ PAVE Technology Company, 2751 Thunderhawk Court, Dayton, OH 45414 USA

${ }^{5}$ BMTI, Parc d'Activités de La Millonne, 117 rue d'Ollioules, 83140 SIX-FOURS - France
} 
Shape of Detection Unit

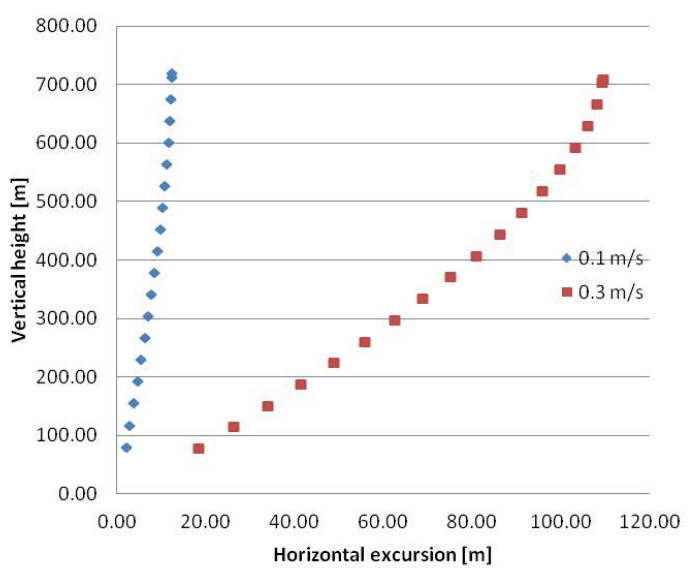

Shape of KM3NeT/ORCA Detection

Unit

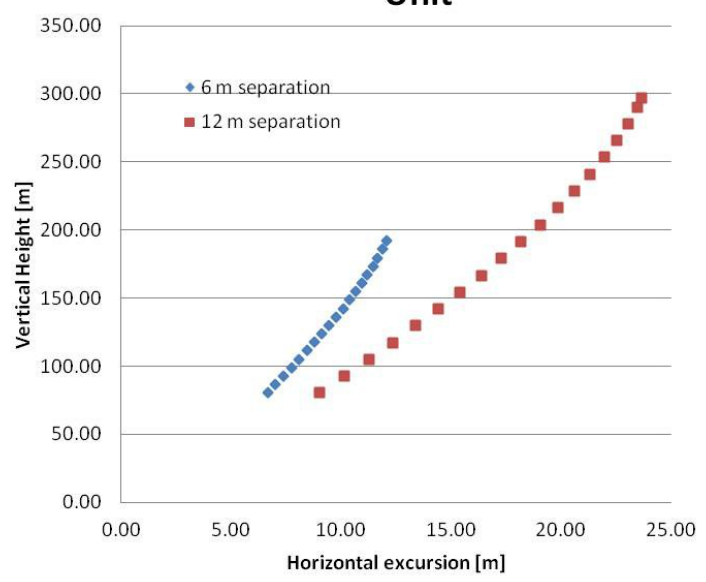

Figure 3: Left:Shape of the KM3NeT/ARCA detection unit for current speeds of $0.1 \mathrm{~m} / \mathrm{s}$ and $0.3 \mathrm{~m} / \mathrm{s}$. Right:Shape of a KM3NeT/ORCA detection unit for $6 \mathrm{~m}$ and $12 \mathrm{~m}$ interDOM distance for a water speed of $0.3 \mathrm{~m} / \mathrm{s}$.

From the maximum total drag and the total buoyancy the minimum negative buoyancy of the anchor can be determined by $M=-1.5 B_{\text {tot }}-2.5 D_{\max }[\mathrm{N}]$. This adds a $50 \%$ safety factor and assumes a coefficient of friction between the anchor and the seabed of 0.6. For the present design $\mathrm{M}=-8000 \mathrm{~N}$.

A major concern in the design is the stretching of the Dyneema ${ }^{\circledR}$ ropes under load. The tension in each rope is at maximum $2000 \mathrm{~N}$ or about $20 \%$ of the yield strength. At this load the manufacturer claims that long term creep will remain negligible over the 15 year lifetime of the detector. The elastic deformation under the $2000 \mathrm{~N}$ load has been measured to be $\frac{\Delta l}{l}=1.0 \times 10^{-5} \mathrm{~F}$, where $\mathrm{F}$ is the tension $(2000 \mathrm{~N})$. As no tension can be sustained by the optical fibers the length of the cable between DOMs must be at least 2\% larger than the length of rope. In the deployment method described in Section Deployment, the length of the rope varies from $35.5 \mathrm{~m}$ to $36.5 \mathrm{~m}$. This in addition to some slack required around the connection to the DOM has led to the cable length of $40 \mathrm{~m}$ between DOMs.

One concern in the design was that the stretching might not be equal in both ropes. The full increase in length over the $750 \mathrm{~m}$ of the detection unit is $15 \mathrm{~m}$. A $1 \%$ difference in stretching of the two ropes would already lead to an unacceptable $15 \mathrm{~cm}$ height difference at the top DOM. Two features were included to counteract this. The ropes are independently stretched at the expected $2000 \mathrm{~N}$ load and marked at equal distances. The DOMs are attached to the ropes using the marks as reference, thus removing the major part of the effect. In addition the connection to the DOM is made over an extended length of $60 \mathrm{~mm}$. If there is a height difference between the two sides of the DOM are different this extended length produces a torque which then translatesinto an additional upward force on the shorter rope and a reduction of the force on the longer. This leads to an increased stretching of the shorter rope and a decreased stretching of the longer rope thus counteracting the height difference. 


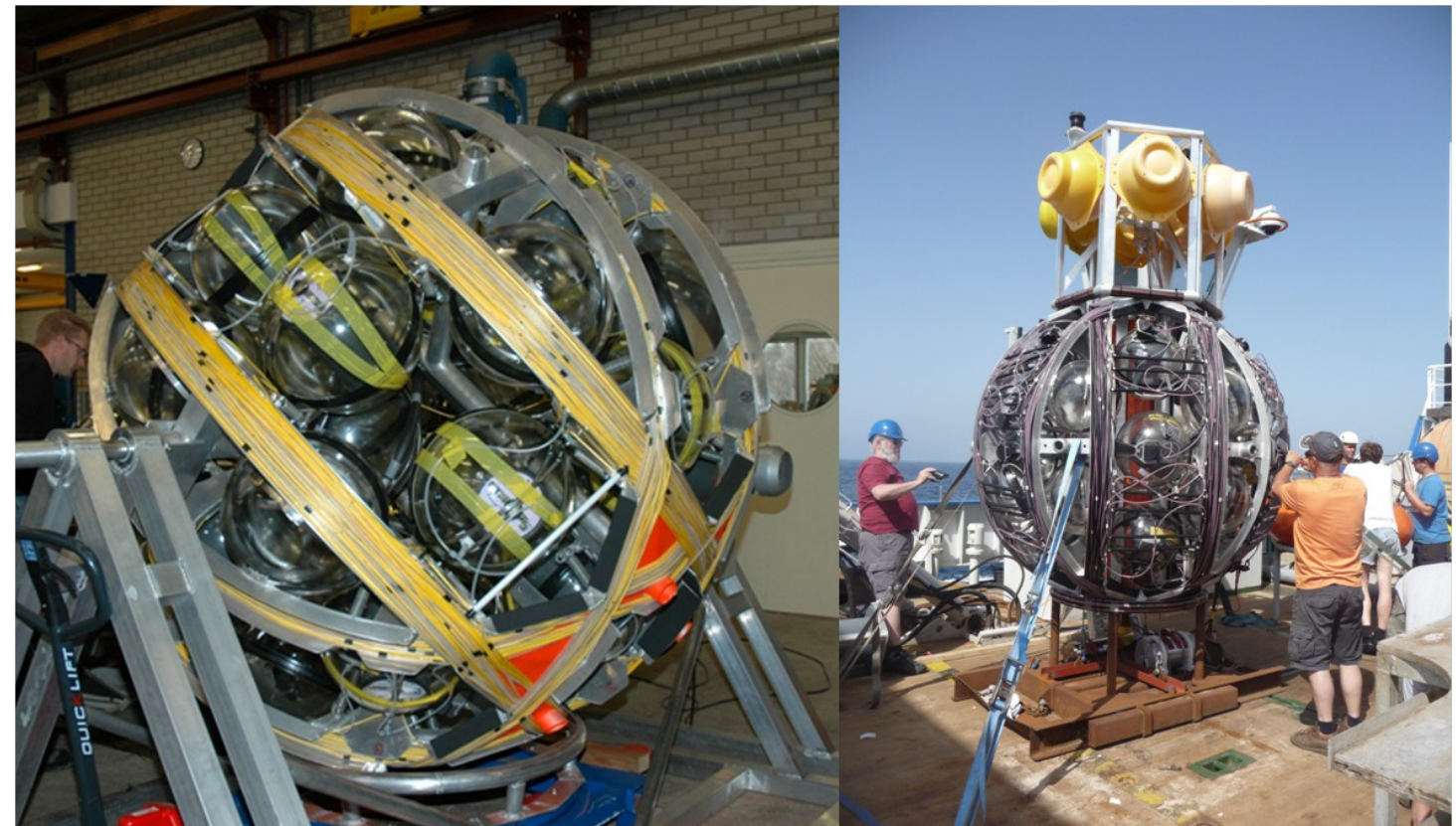

Figure 4: Left: Launcher of Optical Modules (LOM). One of the tracks is visible with cable trays at either side of the receptacles for the (at this point dummy) DOMs. The floatation spheres for recovery of the frame are also visible. Right: Loaded LOM on deck. The flotation frame for the accoustic release is mounted on top.

\section{Deployment}

The placement on the seafloor must be accurate to about $5 \mathrm{~m}$ for ARCA and probably somewhat better for ORCA. This is to provide a reasonable uniformly distributed array without significant holes. This kind of accuracy has been demonstrated in the ANTARES experiment. The method used there of deploying the anchor first and lowering the detection unit while connected to the top of the buoy would require a significant strengthening of the ropes with a consequential increase in drag, as the wave motion during deployment can easily lead to an increase in tension in the ropes of a factor five. The alternative of lowering by the anchor while floating the detection unit above gives a strong risk that the hoist cable and the detection unit would become entangled. The design has therefore been based on placing the detection unit on the sea floor in compacted form and allowing for a subsequent unfurling when the hoist cable has been removed.

The difficulty is then to reduce a $750 \mathrm{~m}$ long detection unit into a manageable, compact form. For this the Launcher for Optical Modules (LOM) has been designed [4]. The LOM, shown in Figure 4, is a spherical structure, with a diameter of $2 \mathrm{~m}$, into which 18 DOMs can be fixed in three "tracks". In addition the cylindrical buoy is housed in the centre of the structure. To assist recovery after the deployment 12 empty 17 inch glass spheres are connected inside the LOM, each with a buoyancy of $250 \mathrm{~N}$. Each DOM is locked in place, suspended from its connection blocks, with a spring-loaded lock that opens at a force of $800 \mathrm{~N}$. Two cable trays are situated next to the DOMs on each of the three tracks. The tracks are at mutual angles of $120^{\circ}$. During loading a DOM is placed in its lock and the LOM is rotated 6 times while the ropes and 


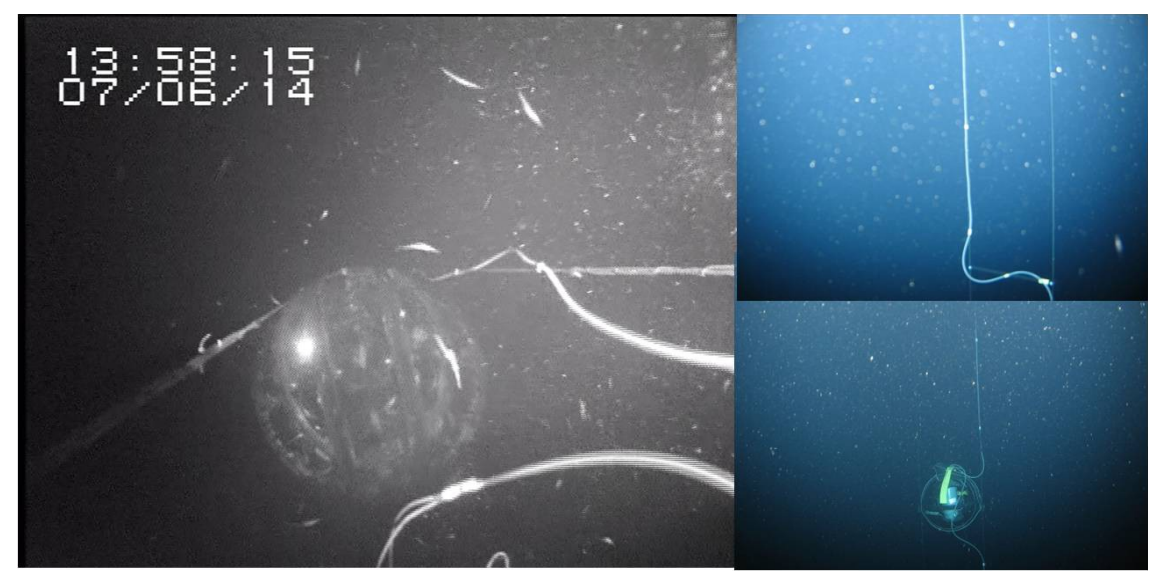

Figure 5: Left:LOM just after release from anchor. One sees the ropes connected to the anchor. The electro-optical cable is also visible(@NIOZ).Right:Pictures taken from the ROV of the cable crossing (top) and DOM (bottom)(CVLIZ).

cable are led into the cable trays. During this exercise spacer bars are inserted between the ropes at alternating intervals of $6 \mathrm{~m}$ and $12 \mathrm{~m}$. These spacers keep the ropes apart, providing a significant torsional stability to the structure and allow for a place where the cable can cross from rope to rope. This is required to balance the drag on both sides and to take up the necessary additional cable length. At each crossing approximately $1.5 \mathrm{~m}$ of cable length is held in place on the spacer bars in such a way that it is extracted when the ropes stretch during deployment.

In this way tension on the cable and the contained optical fibres is avoided. After loading 6 DOMs the LOM is rotated $120^{\circ}$ around the vertical axis and the loading is continued in the next track where again 6 DOMs are loaded. The third track is then selected and after the $18^{\text {th }}$ DOM has been loaded the final $80 \mathrm{~m}$ of cable and ropes is loaded into the third cable tray. The buoy is then inserted and locked in place and connected to the beginning of the ropes. After transfer of the LOM to the anchor the end of the ropes are connected to the anchor. Hoist cables are passed through tubes in the LOM structure and connected to the anchor and to an acoustic release housed in a buoyant frame. When the acoustic release is closed the frame and the LOM are both tightly connected to the anchor

During deployment the full structure of anchor, LOM and acoustic release frame is connected to the ship's crane with a second acoustic release. Flotation is connected to the crane cable about $30 \mathrm{~m}$ above the acoustic release in order to avoid the crane cable becoming entangled with the LOM when the tension in the cable is released as the anchor hits the seabed. When it has been verified (by acoustics) that the anchor and LOM are securely positioned on the sea floor, the cable release is fired and the cable is reeled in. When it is time to release the LOM for unrolling, the release in the buoyant frame is released. The buoyancy in its frame takes it rapidly to the surface, ahead of the released LOM. Through its buoyancy the LOM rises straight at first to clear the hoist cables and then starts to rotate, unrolling the ropes and cable in the process. Figure 5 shows the LOM just after release at the point where the ropes have become taught and the LOM is starting to rotate. The LOM continues to rotate releasing each DOM in turn, the buoyancy of the LOM overcoming the spring load of the locking mechanisms. When all DOMs have exited, the buoy is released from the LOM and the LOM continues on to the 

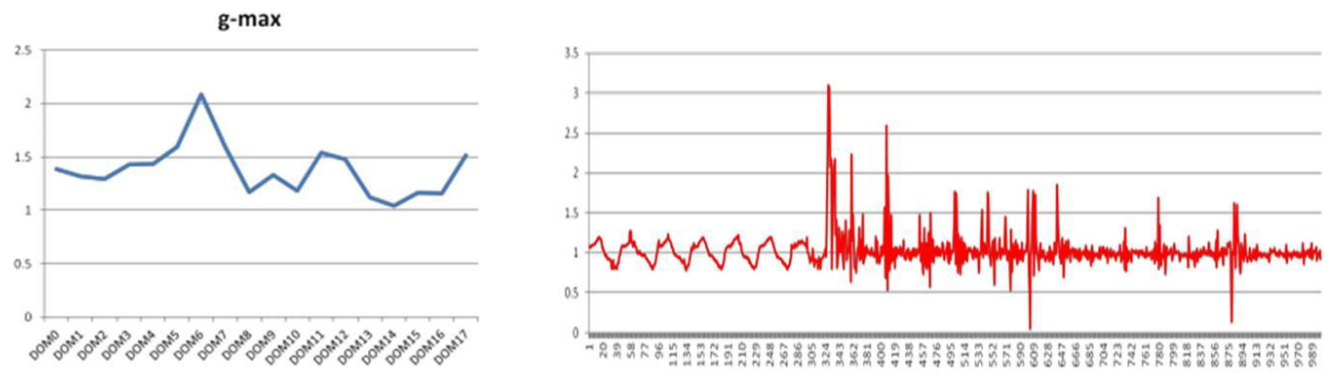

Figure 6: Left: The acceleration experienced by the DOMs during extraction from the LOM. Right: A time sequence of the experienced acceleration for the DOM with the largest peak acceleration. Small oscillations at start are due to the LOM rotation. Peaks following the initial peak at extraction are caused by shaking of the string when subsequent DOMs are extracted.

surface leaving behind a fully unfurled detection unit. Figure 5 also shows pictures of the unfurled detection unit taken by ROV indicating that the cable has been extracted correctly and the DOMs are all horizontal as expected. The deployment technique has been fine tuned and verified during four sea campaigns: two off the coast of Pylos, Greece, and two off the coast of Motril, Spain. Accelerations experienced by the DOMs during extraction are shown in Figure 6. The peak acceleration is $2.2 \mathrm{~g}$.

\section{Conclusions}

A design for the detection unit for the KM3NeT detector has been developed. Its main feature is the minimisation of the hydrodynamic drag. To this end a custom electro-optical cable has been constructed and the mechanical strength is provided by thin Dyneema ${ }^{\circledR}$ cables. To deploy this structure a novel technique of compact deployment with subsequent unfurling of the $750 \mathrm{~m}$ long unit has been developed.

\section{Acknowledgements}

The collaboration gratefully acknowledges the help given by the deck crew, provided to us by the National Institute for Sea Research, NIOZ, in the Netherlands. In addition we thank the ship's crew of the R.V. Pelagia and R.V. Meteor for providing us with safe passage during the four sea campaigns. Finally we acknowledge the Flemish institute for Sea Research,VLIZ, for the use of their ROV including expert crew.

\section{References}

[1] R.Bruijn (for KM3NeT collaboration), Proceedings of this conference.

[2] DSM corporation. http://www.dsm.com/products/dyneema/en_GB/product-technologies/fiber.html.

[3] MIDEL 7131 http://static.mimaterials.com/midel/documents/sales/Midel_Product_Guide_Low_Res_SN.pdf

[4] E. de Wolf et al. (for KM3NeT consortium), Nuclear Instruments and Methods , 725A(2013)241. 